\title{
LEARNING TO MODULATE THE PARTIAL POWERS OF A SINGLE SEMG POWER SPECTRUM THROUGH A NOVEL HUMAN- COMPUTER INTERFACE
}

Ida-Maria Skavhaug ${ }^{1}$, Kenneth R. Lyons ${ }^{1}$, Anna Nemchuk ${ }^{2}$, Shira D. Muroff ${ }^{3}$ and Sanjay S. Joshi ${ }^{1 a}$

${ }^{1}$ Dept. of Mechanical and Aerospace Eng., University of California, Davis, 1 Shields Avenue, Davis, CA 95616.

${ }^{2}$ Dept. of Psychology, University of California, Davis, 1 Shields Avenue, Davis, CA 95616.

${ }^{3}$ Dept. of Human Development, University of California, Davis, 1 Shields Avenue, Davis, CA 95616.

${ }^{a}$ Corresponding author

Ida-Maria Skavhaug: iskavhaug@ucdavis.edu;

Kenneth Lyons: krlyons@ucdavis.edu;

Anna Nemchuk: anemchuk@ucdavis.edu;

Shira Muroff: sdmuroff@ucdavis.edu;

Sanjay Joshi: maejoshi@ucdavis.edu

Page 1 of 25

(C) 2016. This manuscript version is made available under the Elsevier user license http://www.elsevier.com/open-access/userlicense/1.0/ 


\section{ABSTRACT}

New human-computer interfaces that use bioelectrical signals as input are allowing study of the flexibility of the human neuromuscular system. We have developed a myoelectric Human-Computer Interface, which enables users to navigate a cursor to targets through manipulations of partial powers within a single surface electromyography (sEMG) signal. Users obtain two-dimensional control through simultaneous adjustments of powers in two frequency bands within the sEMG spectrum, creating power profiles corresponding to cursor positions. It is unlikely that these types of bioelectrical manipulations are required during routine muscle contractions. Here, we formally establish the neuromuscular ability to voluntarily modulate single-site sEMG power profiles in a group of naïve subjects under restricted and controlled conditions using a wrist muscle. All subjects used the same pre-selected frequency bands for control and underwent the same training, allowing a description of the average learning progress throughout eight sessions. We show that subjects steadily increased target hit rates from $48 \%$ to $71 \%$ and exhibited greater control of the cursor's trajectories following practice. Our results point towards an adaptable neuromuscular skill, which may allow humans to utilize single muscle sites as limited generalpurpose signal generators. Ultimately, the goal is to translate this neuromuscular ability to practical interfaces for the disabled by using a spared muscle to control external machines.

Keywords: Human-Computer Interface; surface electromyogram; frequency bands; motor learning; neuromuscular control. 


\section{INTRODUCTION}

Recent advances in technology have led to the development of a range of different devices enabling communication paths between the human body and machines - collectively referred to as humancomputer interfaces $(\mathrm{HCl})$. Some of these devices utilize the human body's natural bio-electrical signals by translating impulses to external commands. The ultimate purpose of $\mathrm{HCl}$ devices is to restore some of the lost independence for disabled subjects by allowing them to act on their environment through alternative means. $\mathrm{HCl}$ research also allows investigations into the adaptability of the human electrophysiological system and how it may function beyond its original purpose. Here, we report results from 12 subjects learning to use a novel interface that requires the ability to manipulate the surface electromyogram ( $S E M G$ ) recorded from one single muscle site in order to control a cursor in twodimensions simultaneously. It is unlikely that these types of bioelectrical manipulations are required during routine muscle contractions. Our main objective is to formally establish the human body's ability to use our system, and in particular to describe the learning progress as it occurs over multiple testing sessions.

\subsection{Motivation: ELECTROMYOgRAPHy HUMAN-COMPUTER INTERFACES FOR THE DISABLED}

One strong motivation behind $\mathrm{HCl}$ research is to develop control and communication tools for individuals with restricted physical abilities, often caused by accidents or diseases of the central nervous system. Although EMG-driven interfaces are not a viable option for individuals in a 'locked in' state, a large group of even the severely disabled population has limited access to muscles. For example, in the case of high spinal cord injury, these are usually muscles innervated at the brain stem rather than the spinal cord (i.e. head muscles). Other injuries or diseases that cause paralysis may spare muscles on other parts of the body.

Our device is one of many EMG-driven devices for control of external machines that all require subjects to manipulate EMG in different ways. The most commonly known application of EMG occurs in control of powered prostheses (Oskoei \& Hu, 2007; Roche, Rehbaum, Farina, \& Aszmann, 2014; Scheme \& Englehart, 2011; Zecca, Micera, Carrozza, \& Dario, 2002); however, other interfaces also exist that utilize EMG in slightly different ways (Chin, Barreto, Cremades, \& Adjouadi, 2008; Cler \& Stepp, 2015; Hands, 
Larson, \& Stepp, 2014; Larson, Terry, \& Stepp, 2012; Song, Jung, Lee, \& Bien, 2009; Thorp, Larson, \& Stepp, 2014). For example, Cler and Stepp (2015) have developed a novel spelling device in which users navigate and select keys on a keyboard based on SEMG recorded at five different facial sites. EMG has also been used to control powered wheelchairs (Oonishi, Oh, \& Hori, 2010; Song et al., 2009) and in cursor control paradigms, where positions were determined by the contractions recorded on the neck and/or face (Hands \& Stepp, 2014, Thorp et al., 2014, Williams \& Kirsch, 2008).

For control of EMG-driven devices, typically at least one recording site per degree of freedom is needed for continuous control; thus to achieve two-dimensional continuous control of a device, at least two intact recording sites would be employed. Alternatively, a device may be controlled via gesture recognition, in which contractions are analyzed by more sophisticated computational algorithms in order to automatically recognize one of many gestures (De Luca, 1979; Engelhart \& Hudgins, 2003; Scheme \& Englehart, 2011; Zeeca et al., 2002). These gestures are then translated to specific commands. The most novel aspect of our device is that we extract two continuous control-channels from one single signal by simultaneously analyzing the power in two separate frequency bands within the sEMG power spectrum. Essentially, users learn to contract their muscle to create specific power profiles in two bands, which are then translated into cursor coordinates on a computer screen. This motor skill is entirely novel to our subjects and, as it involves an esoteric understanding of signal power properties, subjects appear to approach the task using a trial-and-error learning strategy. From a practical perspective, extracting two control signals from one single sEMG channel allows interfaces with fewer electrodes and associated electronics, which ultimately allows less bodily intrusion for individuals with few available muscle sites.

Our signal processing methods are more closely aligned with some Brain-Computer Interface $(\mathrm{BCl})$ systems (Wolpaw et al., 2004), than traditional EMG based systems. BCls allow characterization of novel electrically-based neurological skills (e.g. Birbaumer, 2006; Wolpaw, Birbaumer, McFarland, Pfurtscheller, \& Vaughan, 2002). Similarly, EMG devices allow characterization of novel electricallybased motor skills involving the recruitment and tuning of hundreds of motor units from one or several underlying muscles which all act cooperatively. Our device aims to co-opt the electrical manipulation 
abilities of the neuromuscular system to control an outside machine. Any associated muscle movement is an unneeded byproduct of the objective to control the machine via the electrical signal.

\subsection{AIMS AND OBJECTIVES}

The primary aims of the current study were to formally establish the ability to intentionally manipulate a single sEMG signal so as to simultaneously place two desired power levels in two separate frequency bands (and thus use our $\mathrm{HCl}$ device) and to describe the learning curve associated with this particular skill. In a previous pilot study, we reported preliminary results with our device in four subjects using the auricularis superior (AS) muscle (located above the ear) to perform a standard cursor-to-target task. The frequency bands used for control were specifically chosen for each subject and training was staged to one target at a time (Perez-Maldonado, Wexler, \& Joshi, 2010). Here, we explore three-target learning from a naïve state in a larger subject sample. Rather than identifying personalized frequency bands, we selected the bands prior to testing to explore the body's ability to adapt to non-personalized bands. Finally, we chose to use the extensor pollicis longus (EPL) muscle, located on the wrist, to investigate the generalization of the neuromuscular ability to muscles innervated at the spinal cord. In our current

study, we observe the performance of 12 naïve subjects over the course of eight one-hour long testing sessions to investigate the progression of performance from the initial encounter with the device.

\section{METHOD}

\subsection{SUBJECTS}

Twelve able-bodied female subjects took part in the experiment (Mean age: 21; range: 18-37), all of which had no prior experience with the myoelectric $\mathrm{HCl}$. The subjects provided written consent as required by the University of California, Davis Institutional Review Board (protocol \#251192) and received psychology course credits per session completed. 


\subsection{ELECTROMYOGRAPHY RECORDING AND SIGNAL PROCESSING}

The processing steps are illustrated in Figure 1. A differential signal is recorded at the muscle site and fed into a computing device (i.e. mobile phone/tablet) via an amplifier (Vernon \& Joshi, 2011). The powers in the two chosen frequency bands are recorded and converted into cursor coordinates on the screen. A cursor-to-target trial begins with a cursor at the rest area in the bottom left hand corner (no contraction), and the user carefully contracts the muscle to continuously guide the cursor to a target on the screen. When a target has been selected, the user confirms the selection by returning the cursor to its rest position in the bottom left corner by relaxing the muscle (hence this feature represents the equivalent of a 'mouse click').

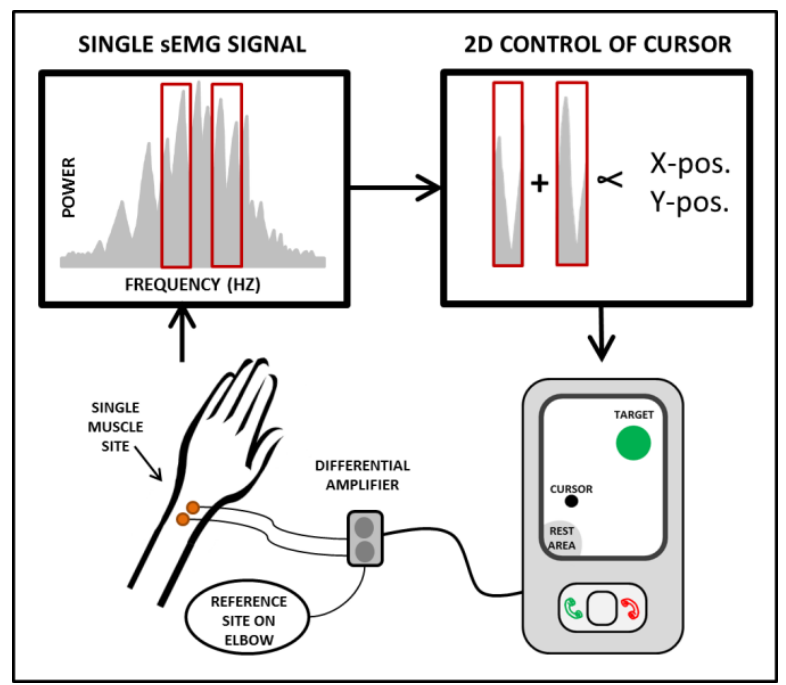

Figure 1. Two sEMG electrodes are placed on the posterior surface of the wrist and record the differential signal which is fed into a standard mobile phone running Android. The power in two separate frequency bands within the single signal is continuously recorded and translated into cursor positions.

We collected a single differential SEMG from the EPL muscle on the subjects' dominant hand with two surface disposable $\mathrm{Ag}-\mathrm{AgCl}$ snap electrodes (6.3 mm diameter) connected to a Motion Labs model Y03 preamplifier with input impedance $>100 \mathrm{M} \Omega, 15-2000 \mathrm{~Hz}$ signal bandwidth and a gain of 300. The electrodes were placed on the posterior surface of the subject's wrist along the axis of the muscle with approximately $1.5 \mathrm{~cm}$ inter-electrode distance (see Figure 2). One additional electrode was placed on the elbow as a reference. Prior to electrode application, the skin was cleaned with an alcohol wipe. The 
snap electrodes were of the type ConMed 1620 Pediatric Huggables Electrodes held in place with Clear NeoDerm tape.

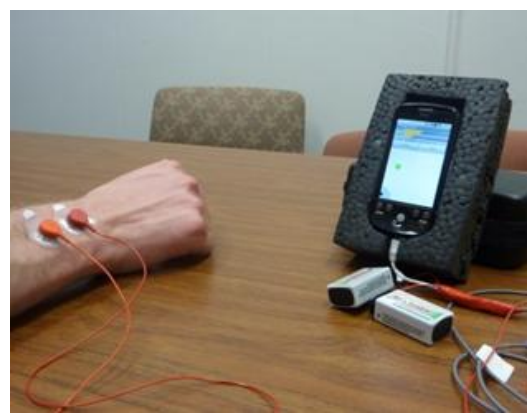

Figure 2. The positioning of the recording electrodes on the extensor pollicis longus. The electrodes were connected to a preamplifier connected to the Android cell phone.

Once the single analog sEMG signal had been measured and filtered $(15-2000 \mathrm{~Hz})$ at the amplifier, the signal was fed into a mobile phone device (HTC MyTouch smartphone running the Android operating system) to create two control channel signals. We sampled the sEMG signal at the standard available sampling rate of $8000 \mathrm{~Hz}$ and then down sampled to effectively sample at $4000 \mathrm{~Hz}$. Power spectrum analysis of the signal at the higher $8000 \mathrm{~Hz}$ sampling rate showed that the SEMG bandwidth for the EPL muscle was well less than $1500 \mathrm{~Hz}$. The first step of signal processing was to compute the total power within the two frequency bands of the single sEMG digitized signal (partial powers). Therefore, the digitized signal was duplicated and then simultaneously filtered using two $2^{\text {nd }}$ order digital Butterworth band pass filters for $80-100 \mathrm{~Hz}$ (Band 1) and $130-150 \mathrm{~Hz}$ (Band 2). These bands were selected following visual inspections of the power spectra of a Maximum Voluntary Contraction (MVC) of the EPL muscle by pilot subjects. The bands appeared to produce comparable powers and were separated by $30 \mathrm{~Hz}$. The bands were the same for every subject.

The equations used to translate partial powers into cursor position are shown in Eqn. 1-4. Total signal power at the output of these filters was simultaneously computed every 0.25 seconds (Power_Band_1(t) and Power_Band_2(t)). The powers were normalized with Max_Power_Band_1 and Max_Power_Band_2 values obtained through a short calibration procedure in which the user 
maintained an apparent comfortable contraction for 5 seconds and maximum power values within this time period were computed for each band. Then, the scaled-normalized powers in each channel were linearly combined using the coefficients shown in Eqns. 1 and 2 to produce an instantaneous cursor position. Finally, in order to reduce jitter, we computed a moving average using the newly computed instantaneous position and the last five instantaneous positions to create the current displayed position (six point moving window average, see Eqns. 3-4). The current transformation equations (Eqn. 1-4) map two positive valued power levels to positions almost exclusively in the first quadrant of a graph (where the rest position occupies the origin). Other transformation equations could conceivably map to other parts of a plane, but have not yet been tested.

The coefficients in Eqns. 1-2 were set such that the user could place the cursor anywhere on the phone screen. The band power values are to some degree correlated and the transformation allows for movement along the axes. Note that if the normalized power in both frequency channels were 1 , then the cursor would be placed on (Xpos=1, Ypos=1), which was defined as the upper right corner of the phone screen. The cursor position could also be scaled according to Effort_Band_1 and Effort_Band_2 parameters that varied from 0-1 and could be adjusted with on-screen commands. These Effort_Band_1 and Effort_Band_2 values allowed more or less muscle contraction effort to be exerted for the same cursor effect. Effort_Band_1 =1 and Effort_Band_2 =1 indicated that full maximal contraction (with reference to max contraction during calibration) would be required to place the cursor in the upper right hand corner of the phone screen. However, these values were nominally set to 0.30 before adjustments to minimize muscle fatigue. If the user's muscle condition and abilities were such that the ability to move the cursor favored one direction over the other, the Effort_Band_1 and Effort_Band_2 could be independently adjusted to equalize control authority in both directions.

The absolute values of Power_Band_1 and Power_Band_2 (partial powers) that were required to hit a particular target varied from subject to subject (and even session to session due to session-to-session calibration) based on Max_Power_Band_1, Max_Power_Band_2, Effort_Band_1, and Effort_Band_2. Note that Max_Power_Band_1, Max_Power_Band_2, Effort_Band_1, and Effort_Band_2 do not change the band locations in frequency space, which were fixed to $80-100 \mathrm{~Hz}$ (Band 1) and 130-150 Hz (Band 2). 
Subjects had to make the average of the last six Power_Band_1s and Power_Band_2s correct to hit the required levels, due to the six point moving window average smoothing procedure (Eqn. 3-4). We used trial and error testing to choose the moving average window size (6 samples). Increasing the moving average window size would cause the cursor to move more smoothly on the screen, but it would also increase the lag in cursor responsiveness, making it harder to temporally map contractions to cursor movement. Decreasing the window size would cause the cursor to move with less lag; however the movements would appear jittery. We chose a six-point moving average window as a satisfactory compromise. In both our internal testing and subject testing, no subjects commented that the lag was noticeable.

These absolute values changed for some of the subjects from session to session due to the need to recalibrate. In order to hit target 1, generally more power (normalized) was necessary in the first band compared to the second band. In order to hit target 3, generally more power was necessary in the second band compared to the first band. Again, the subjects learned to create these profiles as part of the underlying cursor movement capability. They were not aware of these relations.

EQUATION 1.

$$
\begin{gathered}
X_{P O S}(t)=\left[1.75\left(\frac{\text { Power_Band_1 }_{-}(t)}{\text { Max_Power_Band_1 }}\right)\left(\frac{1}{\text { Effort_Band_1 } 1}\right) \cdots\right. \\
\left.-0.75\left(\frac{\text { Power_Band_2 }(t)}{\text { Max_Power_Band_2 }}\right)\left(\frac{1}{\text { Effort_Band_2 }}\right)\right]
\end{gathered}
$$

EQUATION 2.

$$
\begin{aligned}
Y_{P O S}(t) & =\left[-0.75\left(\frac{\text { Power_Band_1 }(t)}{\text { Max_Power_Band_1 }}\right)\left(\frac{1}{\text { Effort_Band_1 }}\right) \cdots\right. \\
& \left.+1.75\left(\frac{\text { Power_Band_2( } t)}{\text { Max_Power_Band_2 }}\right)\left(\frac{1}{\text { Effort_Band_2 }}\right)\right]
\end{aligned}
$$


EQUATION 3

$$
x_{D I S P L A Y}(t)=\frac{1}{6}\left(x_{P O S}(t)+\sum_{n=1}^{5} x_{P O S}(t-n * 0.25 \mathrm{sec})\right)
$$

EQUATION 4

$$
y_{\text {DISPLAY }}(t)=\frac{1}{6}\left(y_{P O S}(t)+\sum_{n=1}^{5} y_{P O S}(t-n * 0.25 \mathrm{sec})\right)
$$

\subsection{EXPERIMENTAL PROTOCOL}

Screen shots of the mobile phone with all three target locations are displayed in Figure 3. The full study protocol was implemented as an application on the mobile phone.

\subsubsection{Setup and Calibration}

The first screen displayed the raw SEMG recording to allow the experimenter to assess the noise level, absence of $60 \mathrm{~Hz}$ interference, and to ensure that the subject was able to manipulate the sEMG recording by contracting the muscle. The subject was then instructed to make three comfortable contractions and the average max power of all three was calculated per band (Max_Power_Band_1; Max_Power_Band_2).
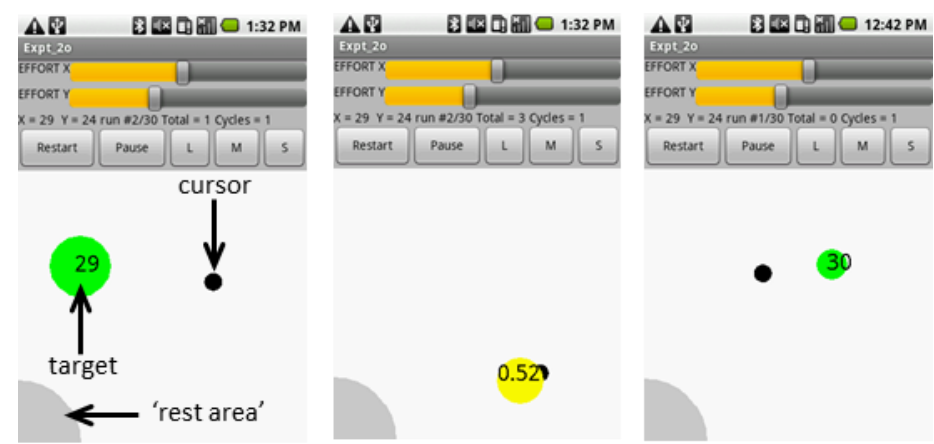

Figure 3. Screen shots of the mobile phone application. The left image shows the cursor and a large-size target in position 1. The number represents the remaining trials of a block. The shaded area is the origin ('rest area'). The middle image shows a medium-size target in position 3, which has been hit and has turned yellow. The number indicates the time-to-target in seconds. The right image shows a small-size target in position 2. 
The final calibration phase allowed the experimenter to assess that the subject could conceivably visit all parts of the phone screen. The final calibration screen displayed the black circular cursor on a white background together with the shaded 'rest area' (origin) and three red-colored screen corners. The subject was instructed to visit all three corners of the screen. When a corner was visited, that corner would change color from red to white to signify that the particular section of the screen had been visited. The subject attempted to visit each of the three corners, and if she appeared to have specific difficulties moving in one direction, then the Effort_Band_1 (dominating x-pos) and Effort_Band_2 (dominating y-pos) could be independently adjusted by the experimenter to compensate for this difficulty. The efforts were adjusted by moving markers on two sliding bars with values ranging from 0 to .50 (set to .30 by default) at the beginning of the calibration procedure. Once this screen was completed, the effort values could not be re-adjusted, and the calibration phase was complete.

\subsubsection{THE 'NULL-TEST' (SESSION 1 ONLY)}

A 'Null-Test' was only administered in the first of the eight sessions and was included to estimate baseline performance. A trial started once the cursor left the shaded rest area in the bottom left corner and ended when either the cursor returned to the rest area or the trial timed out after eight seconds. Subjects were instructed to practice moving the cursor and attempt to cover as much of the phone screen as possible before the trial timed out. Although the Null-Test as a task is not directly comparable to the cursor-to-target task that followed, it was used to provide some indication of the rate at which targets could be successfully hit through random movements.

\subsubsection{CURSOR-TO-TARGET TASK}

A single trial consisted of a user being presented with a target, and then making an attempt to guide the cursor to the target from the rest position within 8-seconds. The targets were presented one at a time as green circles with the number of remaining trials of the block superimposed (Figure 3). There were three target locations and the location was randomly determined for each trial (each was presented an equal number of times). In each session, the subject completed a total of 210 trials divided into seven blocks of 30 trials. The trials timed out after eight seconds unless a target was hit or the cursor returned to the origin. When a target was hit, the target turned yellow and the time-to-target (TT) for that trial was displayed. The subject then relaxed the muscle for the cursor to return to origin and the next trial 
was initiated. The subject was allowed to take breaks anytime he/she felt like it (if this happened in the middle of a block then the experimenter would press the pause button). Typically, short breaks of 1 minute or less were taken between blocks.

There were three target sizes: Large ( $4 \%$ of screen area), Medium ( $2 \%$ of screen area) and Small ( $1 \%$ of screen area). Training progressed from large targets to smaller targets, to reduce early task-anxiety and promote task-mastery later (the complete schedule over all eight sessions is illustrated in Figure 4 as a timeline). However, the first session started with a block of 'Pre-Test' trials in which subjects hit small targets before training on large targets. The very last block of small targets was defined as the 'Post-test' (however from the subjects' point of view this block was not different from the previous one).

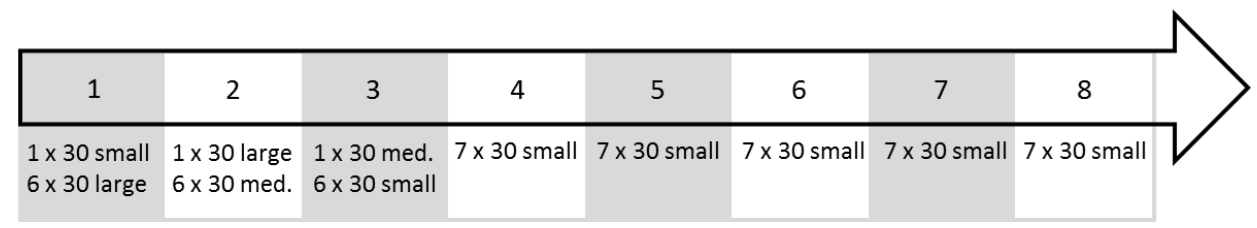

Figure 4. An illustration of the experimental protocol across eight testing sessions: Trials were presented in blocks of 30 with the opportunity for breaks in between.

\section{RESULTS}

Our main performance measures of interest were the proportion of targets hit within the 8 second time limit (success rate) and the time it took to navigate the cursor from the origin to the target (time-totarget; TT). We directly compare small target performance to the Null test, the Pre-Test (first 30 trials of session 1) and the Post-Test (last 30 trials of session 8). We also explored correlation among performance in the Null test, Pre-Test, and the Post-Test. As target size decreased throughout sessions (as learning motivation), we also post-processed the cursor trajectories for the medium and small testing blocks to obtain extrapolated large target success rates and TTs in a separate analysis. We used large target performance across the eight sessions to evaluate learning over time with reference to a constant target size. Finally, as a measure of cursor control, we calculated the percentage of time the cursor spent in the vicinity of the target goal (cursor position distribution analysis including both 
RUNNING HEAD: MYOELECTRIC HUMAN-COMPUTER INTERFACES

successful and unsuccessful trials). This measure was introduced to address intentionality of target hitting (as opposed to random cursor movement).

\subsection{Null-, Pre- ANd Post-Test Performance (small targets)}

Figure 5 shows average success rates (a) and TT (b) on the Null- Pre- and Post-Tests. Paired sample ttests confirmed the impression that Null- and Pre-Tests scores were no different $(p=0.794)$, whereas both Null- $[\mathrm{t}(11)=3.7, \mathrm{p}=0.004]$ and Pre-Tests scores $[\mathrm{t}(11)=3.2, \mathrm{p}=0.009]$ were both significantly lower than Post-Tests. Correspondingly, paired sample t-tests confirmed that Null- and Pre-Test TTs were not significantly different $(p=0.647)$, whereas both Null- $[t(11)=3.6, p=0.004]$ and Pre-Tests scores $[\mathrm{t}(11)=2.7, \mathrm{p}=0.020]$ were significantly higher than Post-Tests TTs. These results show that subjects hit more targets and used less time per trial, following practice.

Next, we performed simple correlational analyses to investigate whether performance on Post-test could be predicted by performance on Null- and/or Pre-Test. Pearson's $r$ is given in

Table 1. For success rates, the analyses indicated a weak positive correlation between both Null- and Pre-Test Scores and weak negative correlations between Null- and Post-Test scores as well as Pre- and Post-Tests scores. For TT, the analyses indicated a negative correlation between Null- and Pre-Test TTs and weak positive correlations between Null- and Pre-Test TTs and Pre- and Post-Test TTs. All the coefficients were however non-significant (all with $p>0.5$ ), suggesting no reliable relationship between scores on the three different tests. 

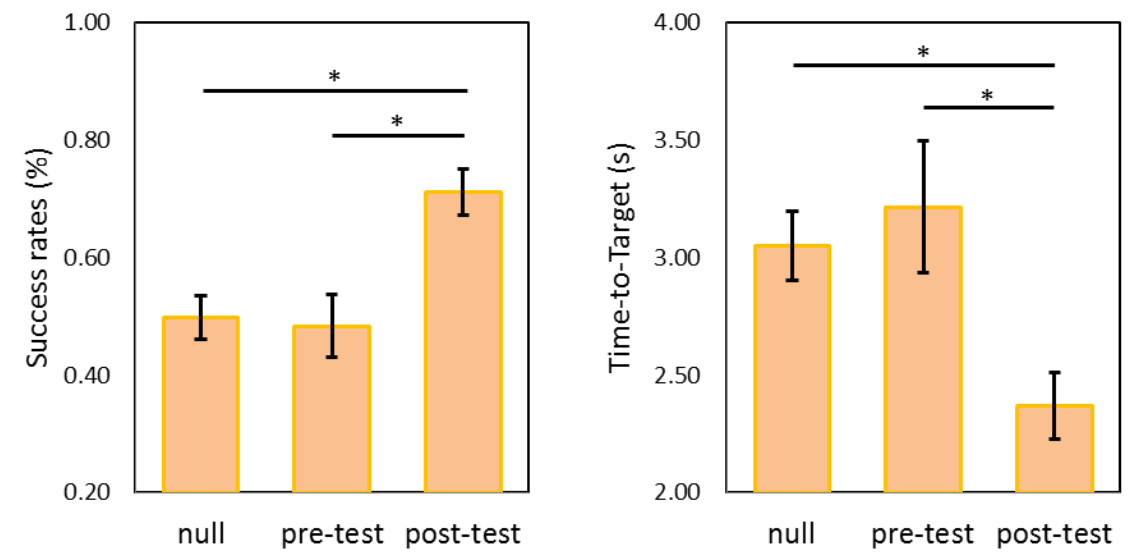

Figure 5. (a) Success rates and (b) TTs on Null-, Pre and Post-Test (small targets). Error bars represents standard error of the mean.

Table 1. Pearson's r correlations of performance measures (for success rates and TTs) between Null-, Pre- and Post-Tests.

\begin{tabular}{|l|cc|cc|}
\hline & \multicolumn{2}{|c|}{ Success Rates } & \multicolumn{2}{c|}{ Time-to-Target } \\
& Null & Pre & Null & Pre \\
\hline Pre & 0.19 & - & -0.25 & - \\
Post & -0.13 & -0.19 & 0.15 & 0.04 \\
\hline
\end{tabular}

\subsection{LEARNING ACROSS EIGHT SESSIONS (LARGE TARGETS)}

Figure 6 shows average success rates (a) and TT (b) across eight testing sessions (large Null-Test scores appear as the first data points on each graph, but were not included in the regression analyses). The success rates increased most rapidly during the first three sessions, possibly reaching a ceiling at around $83 \%$. Mean success rates across subjects were fitted to an exponential function, returning the equation $y=0.84-0.28^{-0.61 x}$ and an adjusted $r^{2}$ of 0.94 . The mean TTs across subjects were similarly fitted to an exponential function, returning the equation $y=2.09+0.41^{-0.36 x}$ and an adjusted $r^{2}$ of 0.87 . 
a

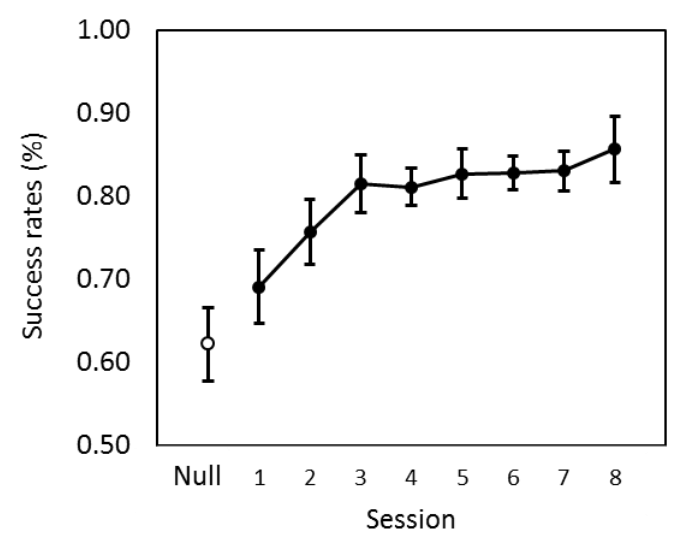

b

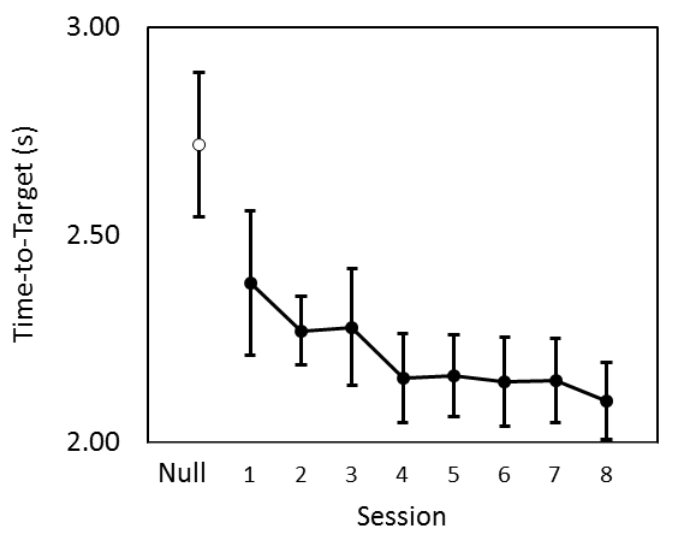

Figure 6. (a) Success rates and (b) TTs across eight testing sessions. First data point on the plots show Null-Test performance (large targets). Error bars represents standard error of the mean.

\subsection{CURSOR POSITION DISTRIBUTION ANALYSIS}

The cursor position distribution analysis was chosen to assess any changes in the amount of time that the cursor spent in the vicinity of the goal target as a function of session number (i.e. practice). The screen was partitioned by lines radiating from the origin out through points half way between adjacent targets. We fitted the mean data points to exponential functions to describe the percentage of time spent in the 'correct' section of the screen when attempting to hit Targets 1, 2 and 3 across the eight sessions (see Figure 7). Note that this analysis includes all trials (both successful and unsuccessful).

All trials for which Target 1 was attempted (Figure 7a), the exponential regression analysis returned the equation $y=0.52-0.61^{-1.32 x}$ and an adjusted $r^{2}$ of 0.77 . For Target 2 (Figure $7 \mathrm{~b}$ ), the analysis returned the equation $y=0.39-0.14^{-0.99 x}$ and an adjusted $r^{2}$ of 0.56. Finally, for Target 3 (Figure 7c), the analysis returned the equation $y=3.32-3.01^{-0.01 x}$ and an adjusted $r^{2}$ of 0.52 . Using one-sample t-tests (with a test value of 0.33 ), we evaluated whether or not the proportion of time spent in the 'correct' section of the screen was significantly different from chance at the first session and at the last. For all Targets, there were no significant differences at session 1, however at session 8 trial time spent in the correct section of the screen was significantly higher than chance (see Table 2). 


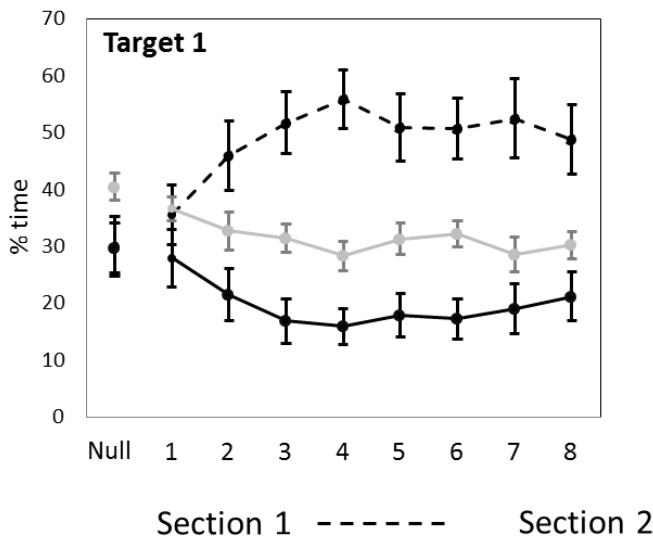

C

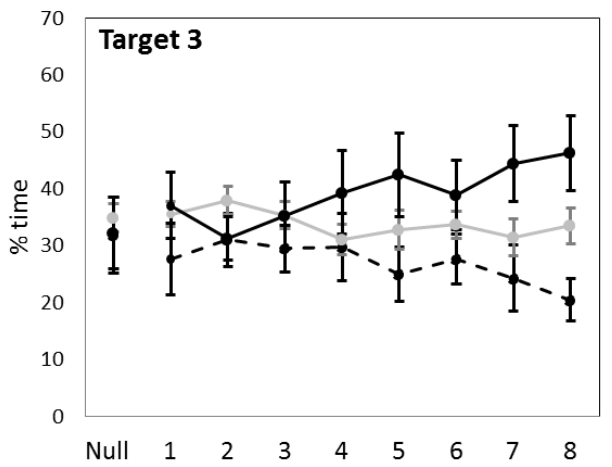

b

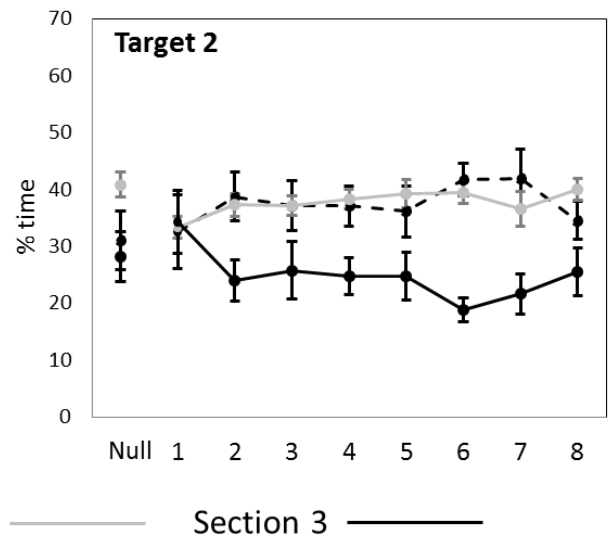

d

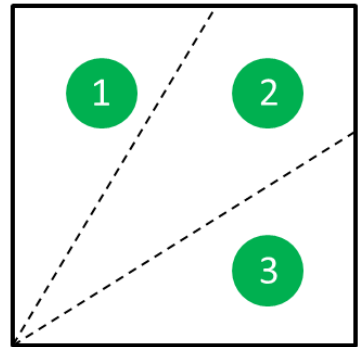

Figure 7. Time spent in each section of the screen when the subject is aiming for (a) Target 1, (b) Target 2, and (c) Target 3. Both successful and unsuccessful trials are included in the data. Error bars represent standard error of the mean. (d) the position of each target and the division of the screen into three sections.

Table 2. Outcome of t-tests comparing the percentage of trial time spent in the correct section of the screen to chance at session 1 and 8 .

\begin{tabular}{|c|cc|}
\hline Goal Target & Session 1 & Session 8 \\
\hline 1 & $t(11)=0.42, p=0.342$ & $t(11)=2.51, p=0.014$ \\
2 & $t(11)=0.05, p=0.519$ & $t(11)=3.47, p=0.002$ \\
3 & $t(11)=0.63, p=0.574$ & $t(11)=1.95, p=0.039$ \\
\hline
\end{tabular}




\section{DISCUSSION}

The current study investigated use of a novel myoelectric Human-Computer Interface $(\mathrm{HCl})$ by 12 naïve subjects across eight one-hour long testing sessions. The experimental task consisted of navigating a cursor to targets, within a time limit of eight seconds, by manipulating one single surface electromyography (sEMG) signal through small contractions of a muscle innervated at the spinal cord (the extensor pollicis longus; EPL). Two-dimensional control was achieved through adjusting the relative powers in two separate frequency bands within the power spectrum, which determined the cursor's position in $x$ - and $y$-space on a mobile phone screen. In contrast to our previous pilot study, the frequency bands used for control were not tailored to the individual, but identified prior to the experiment. Our results show that subjects' target-hitting performance (small targets) improved as a function of experience, from an initial rate of $48 \%$ to $71 \%$ on the eighth session and corresponding timeto-targets steadily decreased. Importantly, as performance improved, the cursor spent a greater proportion of trial time in the vicinity of the relevant target - indicating that subjects were intentionally controlling its movements. Overall, we conclude that cursor control from a single muscle site is a skill that can be learned following relatively short amounts of practice (approximately three hours/600 trials) by a group of subjects. Below we discuss each of our results in detail.

\subsection{IMPROVEMENTS AT POST-TEST}

We included a Null-Test at the very start of our experiment to grossly estimate how many targets subjects could hit by chance when moving the cursor around a blank screen. Performance on the NullTest was compared to performance on the Pre- and Post-Tests which consisted of the first and last thirty trials that the subjects completed in the experiment. This comparison showed that subjects did not do any better on the Pre-Test compared to the Null-Test, suggesting that they had little intentional control of the computer cursor at the beginning of the cursor-to-target test. By the Post-Test at the end of Session 8 , however, there were a significant improvement both in success rates and time-to-target. We also carried out correlational analyses investigating any potential relationship between performance on Null-, Pre- and Post-Test, which could indicate that performance was driven by a general ability to move the cursor more efficiently - an ability that could have been present already at the initial stages of the experiment. We found no evidence of such a correlation; in fact, there was a weak negative correlation between initial performance measures and Post-Test performance. These analyses indicates that a new 
skill has been learned across the eight testing sessions - one that was not driving initial target-hitting ability.

The notion of a 'baseline performance' is difficult to achieve in cursor-to-target paradigms. The Null-Test is only one imperfect measure of pre-training abilities and is likely highly influenced by each subject's interpretation of the instructions given ('cover as much of the screen as possible'). However, it is interesting that Pre-Test scores were not significantly higher than Null-Test scores, a finding that suggests that visually presented targets on the screen made little difference to the ability to successfully direct the cursor to those targets before any training had taken place.

\subsection{A NON-LINEAR LEARNING CURVE}

In addition to improvement from start to finish, we were also interested in describing the learning curve across eight testing sessions. Interestingly, average subject performance improved rapidly throughout the first two testing sessions and then improved only marginally over the remaining sessions. We hypothesize that subjects reached a ceiling at around $83 \%$, where improvement became very difficult. Although negatively accelerating learning curves are often observed in motor learning (Lane, 1987), it was surprising that a ceiling was reached so soon in the experiment. We attribute this to properties of the experimental protocol. In the current experiment, we chose an 8 second time limit, which unarguably made the task more challenging. In addition, subjects were not able to return the cursor to the rest area during target hitting attempts, as this would terminate the trial with a score of 0 (unfortunately this resulted in a certain number of trials being unsuccessful due to 'false starts'). It is therefore difficult to estimate if subjects were likely to improve much beyond the $83 \%$ of large targets hit during the final session, as they may have hit a ceiling defined by the relative difficulty of the task. When designing and testing an interface it is therefore crucial to pay attention to protocol details and know how they correspond to studies reported elsewhere. In the $\mathrm{HCl}$ literature, it is commonly argued that success rates of at least $70 \%$ are necessary for an interface to be practical for communication purposes (Perelmouter \& Birbaumer, 2000); however the proportion of successfully hit targets in any experiment is a product of many variables, such as target size and available trial time. 


\subsection{CURSOR CONTROL}

In all cursor-to-target tasks based on bio-electrical signals, it is difficult to establish with certainty whether the subjects are learning to direct the cursor intentionally or become more proficient at moving the cursor in a 'random fashion' (i.e. covering a larger area of the screen within the allocated trial time). The recorded electrical signals are relatively small and easily prone to random-like jitter and direct paths from the cursor's starting point to the target end-point are rarely observed. The cursor position distribution analyses quantifying the proportion of trial time spent in the 'correct' third section of the screen strongly suggest that subjects are gradually gaining control of the trajectories (importantly, subjects were never instructed to take a direct path to the target or to repeat the same path). Interestingly, subjects overall seemed to find it easier to direct the cursor to Section 1 (upper left) as opposed to Section 3 (lower right) - although at the end of the study they spent above-chance time in the vicinity of the goal target. This may suggest that it took longer to learn how to create the power profiles necessary to place the cursor in the lower right area of the screen (higher relative power in band two). Another surprising observation was that, when aiming for the diagonal target (Target 2), subjects spent almost the same amount of time in Section 1 as in Section 2. This may again have been due to an inherent bias towards Section 1, which the subjects eventually overcame.

\subsection{A NOVEL MOTOR SKILL}

Our study suggests that humans can manipulate surface EMG to serve as limited purpose signal generators for controlling external devices. Subjects were able to modify the partial powers in two frequency bands simultaneously in order to control a cursor in two dimensions. The current study, along with a previous pilot study using the auricularis superior AS muscle of the head, suggest that several different muscles can produce the SEMG signals necessary to volitionally control our device. It is worth noting that the AS muscle is innervated at the brain stem, while the EPL muscle used in this study is innervated at the spinal cord. Future studies may investigate $\mathrm{HCl}$ task performance using a larger muscle responsible for more gross movement (such as the biceps brachii). Similarly, we expect that the recorded signal at any muscle site reflects a combination of activity from the underlying muscle and adjacent muscles, meaning that the relative isolation of a surface muscle from its neighbors may determine how power profiles are produced. 
In the current study, each subject used the same bands to operate the $\mathrm{HCl}$. This was done both to limit experimental variables, and to assess the level of adaptability of the human neuromuscular system. An interesting line of study for $\mathrm{HCl}$ operation is the possibility of using personalized bands to increase performance level. Our previous pilot study used a correlational analysis among possible bands (prior to training) to assign personalized bands. It is unclear whether personalized bands help or hinder device operation. Furthermore, it is still an open question how best to personalize the bands. A future study could address these issues.

One interesting observation made during the experimental sessions was that subjects were often inclined to believe that the cursor would move in the direction the thumb was pointing (like a joystick) despite being explicitly told there was no known correlation between overt muscle movement and cursor output. Using a muscle which contributes to movement of a body part with a large repertoire of natural movements may have distracted some subjects from learning the relationship between the contractions and the cursor positions due to active testing of inaccurate hypotheses.

Any device that relies on muscle activity can potentially cause fatigue with use. Fatigue is a topic that we have investigated in a study run in parallel with the current study and thus far our results show that it is not a concern even following three hours of continuous use of our device using the EPL or AP muscles (unpublished). Presumably this is due to the low levels of contraction required to control the cursor.

Although cursor navigation performance increased as a function of practice, it is worth noting that the subjects were unable to articulate what they did differently at the start and the end of the study - and the nature of the skill learnt remains largely unknown. The creation of power profiles necessitates an extraordinary ability of the central nervous system to fine-tune motor-unit firing and recruitment, causing systematic changes in the powers of the chosen frequency bands - but there are at least two different ways that subjects may be using this ability: i) they may be creating distinct power profiles, which map directly on to the three possible target locations on the screen, which represents a limited skill, or; ii) they may be learning to adjust powers continuously to navigate the cursor to any point on the screen, which represents a much more sophisticated ability. The fact that required power profiles 
changed session to session (as in the case of recalibration) and sensor placement unavoidably shifted slightly, but performance still improved, may point to a more generalizable skill. Future studies should aim to address the exact quality of the skill involved as it will highlight the potential of practical implementations of the myoelectric $\mathrm{HCl}$.

\subsection{CONCLUSION}

We report performance on a myoelectric Human-Computer Interface by twelve naïve subjects across eight one-hour long testing sessions and show that subjects can rapidly learn to create the necessary sEMG power profiles within one signal to guide a mouse cursor to targets on a screen. This novel method of extracting multiple control channels from one single signal requires the acquisition of a very unusual motor skill, and highlights the flexibility of the human neuromuscular system. 


\section{ACKNOWLEDGEMENTS}

We thank all our subjects for taking part in this study. The project was funded by The Hartwell

Foundation and through NSF Grant 0966963. The authors declare no competing financial interest. 


\section{REFERENCES}

Birbaumer, N. (2006). Breaking the silence: Brain-computer interfaces (BCI) for communication and motor control. Psychophysiology, 43(517-532).

Chin, C. A., Barreto, A., Cremades, J. G., \& Adjouadi, M. (2008). Integrated electromyogram and eye-gaze tracking cursor control system for computer users with motor disabilities. Journal of Rehabilitation Research \& Development, 45(1), 161-174.

Cler, M. J., \& Stepp, C. E. (2015). Discrete vs. Continuous Mapping of Facial Electromyography for Human-Machine-Interface Control: Performance and Training Effects. IEEE Transactions on Neural Systems and Rehabilitation Engineering, in press.

De Luca, C. (1979). Physiology and Mathematics of Myoelectric Signals. IEEE Transactions on Biomedical Engineering, 26(6), 313-325.

Engelhart, K., \& Hudgins, B. A. (2003). A robust, real-time control scheme for multifunction myoelectric control. IEEE Transactions on Biomedical Engineering, 40, 848-854.

Hands, G. L., Larson, E., \& Stepp, C. E. (2014). Effects of augmentative visual training on audio-motor mapping. Human movement science, 35(145-155).

Hands, G. L., \& Stepp, C. E. (2014). Effect of Age on Human-Computer Interface Control Via Neck Electromyography. Interacting with Computers, in press.

Lane, N. E. (1987). Skill qcquisition rates and patterns: issues and training implications. New York: Springer-Verlag.

Larson, E., Terry, H. P., \& Stepp, C. E. (2012). Audio-visual feedback for electromyographic control of vowel synthesis. 34th Annual International Conference of the IEEE Engineering of Medicine and Biology Society, San Diego, CA.

Oonishi, Y., Oh, S., \& Hori, Y. (2010). A New Control Method for Power-Assisted Wheelchair Based on the Surface Myoelectric Signal. IEEE Transactions on industrial electronics, 57(9), 3191-3196. 
RUNNING HEAD: MYOELECTRIC HUMAN-COMPUTER INTERFACES

Oskoei, M. A., \& Hu, H. (2007). Myoelectric control systems - a survey. Biomedical Signal Processing and Control, 2, 275-294.

Perez-Maldonado, C., Wexler, A. S., \& Joshi, S. S. (2010). Two-dimensional cursor-to-target control from single muscle site sEMG signals. IEEE Transactions on Neural Systems and Rehabilitation Engineering, 18(2), 203-209.

Roche, A. D., Rehbaum, H., Farina, D., \& Aszmann, O. C. (2014). Prosthetic Myoelectric Control Strategies: A Clinical Perspective. Current Surgery Reports, 2(44).

Schalk, G., Miller, K. J., Anderson, N. R., Wilson, J. A., Smyth, M. D., Ljemann, J. G., . . Leuthardt, E. C. (2008). Two-dimensional movement control using electrocorticographic signals in humans. Journal of Neural Engineering, 5(1), 75-84.

Scheme, E., \& Englehart, K. (2011). Electromyogram pattern recognition for control of powered upperlimb prostheses: state of the art and challenges for clinical use. Journal of Rehabilitation Research \& Development, 48(6), 643-660.

Song, J., Jung, J., Lee, S., \& Bien, Z. (2009). Robust EMG pattern recognition to muscular fatigue effect for powered wheelchair control. Journal of Intelligent \& Fuzzy Systems, 20, 3-12.

Thorp, E. B., Larson, E., \& Stepp, C. E. (2014). Combined Auditory and Vibrotactile Feedback for HumanMachine-Interface Control. IEEE Transactions on Neural Systems and Rehabilitation Engineering.

Vernon, S., \& Joshi, S. S. (2011). Brain-Muscle-Computer Interface: Mobile phoneprototype development. IEEE Transactions on Information Technology in Biomedicine, 15(4), 531-538.

Williams, M. R., \& Kirsch, R. F. (2008). Evaluation of head orientation and neck muscle EMG signals as command inputs to a human-computer interface for individuals with high tetraplegia. IEEE Transactions on Neural Systems and Rehabilitation Engineering, 16, 485-496.

Wolpaw, J. R., Birbaumer, N., McFarland, D. J., Pfurtscheller, G., \& Vaughan, T. M. (2002). Braincomputer interfaces for communiaction and control. Clinical Neurophysiology, 113(6), 767-791.

Wolpaw, J. R., McFarland, D. J., \& Bizzi, E. (2004). Control of a two-dimentional movement signal by a noninvasive Brain-Computer Interface in humans. Proceedings of the National Academy of Sciences of the United States of America, 101(52), 17849-17854. 
RUNNING HEAD: MYOELECTRIC HUMAN-COMPUTER INTERFACES

Zecca, M., Micera, S., Carrozza, M. C., \& Dario, P. (2002). Control of multifunctional prosthetic hand by processing the electromyographical signal. Critical Reviews in Biomedical Engineering, 30, 459-485.

Page 25 of 25 